\title{
HOLOMORPHIC RANK-2 VECTOR BUNDLES ON NON-KÄHLER ELLIPTIC SURFACES
}

\author{
VASILE BRÎNZĂNESCU AND RUXANDRA MORARU \\ Dedicated to Professor F. Hirzebruch for his 75th birthday.
}

\begin{abstract}
The existence problem for vector bundles on a smooth compact complex surface consists in determining which topological complex vector bundles admit holomorphic structures. For projective surfaces, Schwarzenberger proved that a topological complex vector bundle admits a holomorphic (algebraic) structure if and only if its first Chern class belongs to the Neron-Severi group of the surface. In contrast, for non-projective surfaces there is only a necessary condition for the existence problem (the discriminant of the vector bundles must be positive) and the difficulty of the problem resides in the lack of a general method for constructing non-filtrable vector bundles. In this paper, we close the existence problem in the rank-2 case, by giving necessary and sufficient conditions for the existence of holomorphic rank-2 vector bundles on non-Kähler elliptic surfaces.
\end{abstract}

\section{INTRODUCTION}

In this paper, we study the existence of holomorphic vector bundles on nonKähler elliptic surfaces; their classification and stability properties are discussed in BrMo1, BrMo2]. Let $X$ be a smooth compact complex surface. The existence problem for vector bundles on $X$ consists in determining which topological complex vector bundles admit holomorphic structures, or equivalently, in finding all triples $\left(r, c_{1}, c_{2}\right)$ in $\mathbb{N} \times N S(X) \times \mathbb{Z}$ for which there exists a rank- $r$ holomorphic vector bundle on $X$ with Chern classes $c_{1}$ and $c_{2}$. For projective surfaces, Schwarzenberger [S] proved that any triple $\left(r, c_{1}, c_{2}\right)$ in $\mathbb{N} \times N S(X) \times \mathbb{Z}$ comes from a rank- $r$ holomorphic (algebraic) vector bundle. In contrast, for non-projective surfaces, there is a natural necessary condition for the existence problem $[\mathrm{BaL}, \mathrm{BrF}, \mathrm{LeP}$ :

$$
\Delta\left(r, c_{1}, c_{2}\right):=\frac{1}{r}\left(c_{2}-\frac{r-1}{2 r} c_{1}^{2}\right) \geq 0 .
$$

One can always construct filtrable bundles by using extensions of coherent sheaves; in fact, on a non-algebraic surface $X$, there exists a filtrable rank- $r$ holomorphic vector bundle $E$ with Chern classes $c_{1}$ and $c_{2}$ if and only if its discriminant $\Delta(E)$ satisfies the inequality

$$
\Delta(E):=\Delta\left(r, c_{1}, c_{2}\right) \geq m\left(r, c_{1}\right),
$$

The first author was partially supported by grant CNCSIS 33518/2002 and by EURROMMAT Programme FP5/2000-2003.

2000 Mathematics Subject Classification. 14J60 (primary), 14D22, 14F05, 14J27, 32J15 (secondary). 
where

$$
m\left(r, c_{1}\right):=-\frac{1}{2 r} \max \left\{\sum_{1}^{r}\left(\frac{c_{1}}{r}-\mu_{i}\right)^{2}, \mu_{1}, \ldots, \mu_{r} \in N S(X), \sum_{1}^{r} \mu_{i}=c_{1}\right\}
$$

(see $\mathrm{BaL}, \mathrm{BrF}, \mathrm{LeP})$. Therefore, the only unknown situations occur for bundles of rank greater than one that have a discriminant in the interval $\left[0, m\left(r, c_{1}\right)\right)$; vector bundles with such discriminants will, of course, be non-filtrable and the difficulty of the problem resides in the lack of a general method for constructing non-filtrable bundles. One is thus compelled to focus on particular classes of surfaces, to find specific construction methods.

The existence of bundles on non-projective surfaces is, in general, still an open question, which has been completely settled only in the case of primary Kodaira surfaces $\mathrm{ABrTO}$. For rank-2 holomorphic vector bundles, the problem has been solved for complex 2-tori [To, as well as for surfaces of class VII and K3 surfaces TTo; since the method used in TTo (Donaldson polynomials) seems to also work for (non-algebraic) Kähler elliptic surfaces, only the case of general non-Kähler elliptic surfaces remains. In this article, we close the existence problem in the rank2 case, by giving necessary and sufficient conditions for the existence of holomorphic rank-2 vector bundles on non-Kähler elliptic surfaces.

Recall that a surface is said to be elliptic if it admits a holomorphic fibration over a curve with generic fibre an elliptic curve; for instance, non-Kähler elliptic surfaces are given by holomorphic fibrations without a section whose smooth fibres are isomorphic to a fixed elliptic curve. For vector bundles on any elliptic fibration $\pi: X \rightarrow B$, restriction to a fibre is a natural operation: there exists a divisor in the relative Jacobian $J(X)$ of $X$, called the spectral curve or cover of the bundle, that encodes the isomorphism class of the bundle over each fibre of $\pi$. This divisor is an important invariant of bundles on elliptic fibrations, which has proven very useful in their study (see [F1, FM] [FMW, BJPS [D]) for projective fibrations, DOPW1, DOPW2 for Calabi-Yau threefolds without a section, and [BH, Mo, T] for non-Kähler fibre bundles). The spectral construction presented in this paper is a modification of the Fourier-Mukai transform for certain elliptic fibrations without a section, which will be used in BrMo1 to define a twisted Fourier-Mukai transform that is specific to non-Kähler elliptic surfaces.

The paper is organised as follows. We begin by presenting and proving some topological and geometrical properties of non-Kähler elliptic surfaces; in particular, we show that if $\pi: X \rightarrow B$ is such a surface, then the restriction of any vector bundle on $X$ to a smooth fibre of $\pi$ always has degree zero. Unlike the algebraic case [FM], the description of line bundles on non-Kähler elliptic surfaces is not straightforward; indeed, even though these surfaces have very few divisors (they are given by the fibres of $\pi$ ), there exist many line bundles on them. Nonetheless, we are able to establish a correspondence between line bundles on a non-Kähler elliptic surface and sections of its relative Jacobian; this follows from results of Br1, Br2, Br3, BrU] regarding the Neron-Severi and Picard groups of these surfaces. In the third section, we extend the spectral construction of $\mathrm{BH}, \mathrm{Mo}$ to the case of holomorphic rank-2 vector bundles on non-Kähler elliptic surfaces. Finally, the last section contains the proof of the existence theorems. 
We end by noting that the techniques developed here and in BrMo1, BrMo2 can be used to solve existence and classification problems for holomorphic vector bundles of arbitrary rank on non-Kähler elliptic and torus fibrations.

\section{LINE BUNDLES}

Let $X \stackrel{\pi}{\rightarrow} B$ be a minimal non-Kähler elliptic surface, with $B$ a smooth compact connected curve; it is well-known that $X \stackrel{\pi}{\rightarrow} B$ is a quasi-bundle over $B$, that is, all the smooth fibres of $\pi$ are isomorphic to a fixed elliptic curve $T$ and the singular ones (if any) are isogeneous to multiples of $T$ (see [Kod, $\mathrm{Br} 3$ ). We begin by presenting several topological and geometric properties of these surfaces.

Let $T^{*}$ denote the dual of $T$ (we fix a non-canonical identification $T^{*}:=\operatorname{Pic}^{0}(T)$ ). In this case, the Jacobian surface associated to $X \stackrel{\pi}{\rightarrow} B$ is simply

$$
J(X)=B \times T^{*} \stackrel{p_{1}}{\rightarrow} B
$$

(see, for example, $\mathrm{Kod}, \mathrm{BVP}, \mathrm{Br1}$ ) and the surface is obtained from its relative Jacobian by a finite number of logarithmic transformations $\mathrm{Kod}, \mathrm{BVP} \mid \mathrm{BrU}]$. Also, if $X$ has multiple fibres $T_{1}, \ldots, T_{r}$, with corresponding multiplicities $m_{1}, \ldots, m_{r}$, then its canonical bundle is given by

$$
K_{X}=\pi^{*} K_{B} \otimes \mathcal{O}_{X}\left(\sum_{i=1}^{r}\left(m_{i}-1\right) T_{i}\right) .
$$

Finally, we the following identification $\mathrm{Br}$ 1, $\mathrm{Br} 2, \mathrm{BrU}]$ :

$$
N S(X) / \operatorname{Tors}(N S(X)) \cong \operatorname{Hom}\left(J_{B}, \operatorname{Pic}^{0}(T)\right),
$$

where $N S(X)$ is the the Neron-Severi group of the surface and $J_{B}$ denotes the Jacobian variety of $B$; the torsion of $H^{2}(X, \mathbb{Z})$ is generated by the classes of the fibres (both smooth and multiple). In the remainder, the class modulo $\operatorname{Tors}\left(H^{2}(X, \mathbb{Z})\right)$ of an element $c \in H^{2}(X, \mathbb{Z})$ will be denoted $\widehat{c}$. Given these considerations, we have:

Lemma 2.1. Let $X \stackrel{\pi}{\rightarrow} B$ be a non-Kähler elliptic surface.

(i) If $c \in N S(X)$, then $\pi_{*}(c)=0$ and $c \cdot \beta=0$ for all $\beta \in \operatorname{Tors}\left(H^{2}(X, \mathbb{Z})\right)$.

(ii) For any element $c \in N S(X), c^{2}=-2 \operatorname{deg}(\widehat{c})$.

Proof. The lemma is certainly true for torsion classes. Let us then assume that $c \notin \operatorname{Tors}(N S(X))$ and choose a line bundle $L$ on $X$ with first Chern class $c$. Then $\widehat{c} \neq 0$ and, by fixing a base-point in $B$, the cohomology class $\widehat{c}$ can be considered as a covering map $\widehat{c}: B \rightarrow P i c^{0}(T)$ such that

$$
\widehat{c}^{-1}\left(\lambda_{0}\right)=\left\{b \in B|L|_{F_{b}} \simeq \lambda_{0}\right\} .
$$

Since $\widehat{c} \neq 0$, we have $\widehat{c}^{-1}\left(\mathcal{O}_{T}\right) \neq B$. Therefore, the stalk of $\pi_{*} L$ is zero at the generic point in $B$ and the direct image sheaf $\pi_{*} L$ vanishes; furthermore, the higher direct image sheaf $R^{1} \pi_{*} L$ is a torsion sheaf supported on $\widehat{c}^{-1}\left(\mathcal{O}_{T}\right)$. In particular, $\pi ! L=-R^{1} \pi_{*} L$ and, by Grothendieck-Riemann-Roch, the pushdown $\pi_{*}(c)$ is equal to the rank of the torsion sheaf $R^{1} \pi_{*} L$, which is zero. Let $\beta$ be a generator of the torsion of $H^{2}(X, \mathbb{Z})$. The class of $\beta$ is then the first Chern class of a sheaf on $X$ of the form $\mathcal{O}(F)$, where $F$ is a fibre of $\pi$ of multiplicity $m \geq 1$. Consequently, the pullback to $X$ of the positive generator $h$ of $H^{2}(B, \mathbb{Z})$ is equal to $c_{1}(\mathcal{O}(m F))$ and, by the Projection formula, we have

$$
m(c \cdot \beta) h=\pi_{*}\left(c \cdot \pi^{*} h\right)=\pi_{*}(c) \cdot h=0,
$$


that is, $c \cdot \beta=0$, proving (i). Combining the results of (i) with GrothendieckRiemann-Roch, we obtain $c_{1}\left(R^{1} \pi_{*} L\right)=-\frac{1}{2} c^{2} \cdot h$. Hence, the degree of the map $\widehat{c}$ is equal to $\#\left(\widehat{c}^{-1}\left(\mathcal{O}_{T}\right)\right)=-\frac{1}{2} c^{2}$ and we are done.

Lemma 2.2. Let $\pi: X \rightarrow B$ be a non-Kähler elliptic surface and $\mathcal{L}$ a line bundle on $X$. The restriction of $\mathcal{L}$ to any smooth fibre of $\pi$ has degree zero.

Proof. Let $m_{1} T_{1}, m_{2} T_{2}, \ldots, m_{\ell} T_{\ell}$ be the multiple fibres of $\pi$ and set $b_{i}=\pi\left(T_{i}\right)$. Denote $m$ the least common multiple of $m_{1}, m_{2}, \ldots, m_{\ell}$ and choose a non-negative integer $e$ such that $m$ divides $\ell+e$; next, take distinct points $b_{\ell+1}, \ldots, b_{\ell+e}$, which are different from $b_{i}, i=1, \ldots, \ell$, and fix a point $b$ with $T_{b}$ smooth. Then, there exists at least one line bundle $M$ on $B$ with the property that

$$
M^{\otimes m} \cong \mathcal{O}_{B}\left(b_{1}+\ldots+b_{\ell+e}\right)
$$

such a line bundle defines an $m$-cyclic covering $\varepsilon: B^{\prime} \rightarrow B$ that is totally ramified at $b_{1}, \ldots, b_{\ell+e}$ (see [BVP], Chapter I, Lemma 17.1). By Lemma 3.18 in Br3, there exists a principal $T$-bundle $\pi^{\prime}: X^{\prime} \rightarrow B^{\prime}$ and an $m$-cyclic covering $\psi: X^{\prime} \rightarrow X$ over $\varepsilon: B^{\prime} \rightarrow B$; let $\tilde{T}$ be a connected component of $\psi^{-1}\left(T_{b}\right)$. Then $\tilde{T}$ is a fibre of $\pi^{\prime}$ and the restriction $\tilde{T} \rightarrow T_{b}$ of $\psi$ is an isomorphism. Therefore, we have

$$
c_{1}\left(\left.\mathcal{L}\right|_{T_{b}}\right)=c_{1}\left(\left.\psi^{*}(\mathcal{L})\right|_{\tilde{T}}\right)=0,
$$

because $\pi^{\prime}: X^{\prime} \rightarrow B^{\prime}$ is a principal elliptic bundle [Br3. T].

Remark. Similar results are stated in ABrTo, T] for non-Kähler principal elliptic bundles, that is, non-Kähler elliptic surfaces without multiple fibres.

Referring to Lemma 2.2 and $[\mathrm{BrU}]$, we can therefore associate to any line bundle $\mathcal{L}$ on $X$ a holomorphic mapping $\varphi: B \rightarrow T^{*}$ such that

$$
\left.\mathcal{L}\right|_{T_{b}}=\varphi(b),
$$

for any smooth fibre $T_{b}$, that is, a section of $J(X)=B \times T^{*}$. Conversely, one can associate to every section of $J(X)$ a line bundle on $X$, as stated in:

Proposition 2.3. Let $\pi: X \rightarrow B$ be a non-Kähler elliptic surface, with general fibre $T$, and $J(X)=B \times T^{*}$ be the associated Jacobian surface of $X$. Then:

(i) For any section $\Sigma \subset J(X)$, there exists a line bundle $\mathcal{L}$ on $X$ whose restriction to every smooth fibre $T_{b}$ is the same as the line bundle $\Sigma_{b}$ of degree zero on $T=T_{b}$.

(ii) The set of all line bundles on $X$ that restrict, on every smooth fibre of $\pi$, to the line bundle of degree zero determined by the section $\Sigma$ is a principal homogeneous space over $P_{2}$, where $P_{2}$ is the subgroup of line bundles on $X$ generated by $\pi^{*} P i c(B)$ and the $\mathcal{O}_{X}\left(T_{i}\right)^{\prime}$ '

Proof. Choose a general point $b \in B$ with $T_{b}$ smooth and consider the natural restriction morphism $r: \operatorname{Pic}(\mathrm{X}) \rightarrow \operatorname{Pic}\left(\pi^{-1}(\mathrm{~b})\right)=\operatorname{Pic}(\mathrm{T})$. Let $\left(P_{j}\right)$ be the filtration of $\operatorname{Pic}(X)$ defined by

$$
P_{0}=\operatorname{Pic}(\mathrm{X}), P_{1}=\operatorname{Ker}(\mathrm{r}) \text {, and } P_{2} \text {. }
$$

Set $N(X):=P_{0} / P_{1}$ and $\tilde{N}(X):=\left\{c_{1}(L) \mid L \in N(X)\right\}$. Referring to [Br1] and BrU, we have $\tilde{N}(X)=0$ and

$$
N S(X) / \operatorname{Tors} N S(X) \cong \operatorname{Hom}\left(J_{B}, T^{*}\right) \cong P_{1} / P_{2} .
$$


Consequently, $N(X) \subset \operatorname{Pic}^{0}(T)$. Since any line bundle in $\operatorname{Pic}^{0}(T)$ is invariant by translations, we obtain

$$
N(X)=\operatorname{Pic}^{0}(T)
$$

by Lemma 2.2 and $[\mathrm{BrU}]$. Let $\lambda=\Sigma_{b} \in T^{*}$ and let $\Sigma^{\lambda}$ be the constant section $B \times \lambda \subset J(X)$. Following the construction in $\mathrm{BrU}$, the line bundle $\lambda \in T^{*}$ extends to a line bundle $\mathcal{L}^{\lambda}$ on $X$ that corresponds to the constant section $\Sigma^{\lambda}$. Let $B_{0}$ be the zero section of $J(X)$. Given the identification $P_{1} / P_{2} \cong \operatorname{Hom}\left(J_{B}, T^{*}\right)$, there exists a line bundle $\mathcal{L}_{1}$ in $P_{1}=\operatorname{Ker}(r)$ whose corresponding element in $\operatorname{Hom}\left(J_{B}, T^{*}\right)$ is a section that is linearly equivalent to $\Sigma-\Sigma^{\lambda}+B_{0}$ (look at the addition law of the group $\left.\operatorname{Hom}\left(J_{B}, T^{*}\right)\right)$. The line bundle $\mathcal{L}=\mathcal{L}_{1} \otimes \mathcal{L}^{\lambda}$ is then such that its restriction to every smooth fibre $T_{b}$ is the same as the line bundle $\Sigma_{b} \in T^{*}$, proving (i). If the line bundles $\mathcal{L}^{\prime}$ and $\mathcal{L}$ on $X$ both have the above property, then by the same isomorphism, $\mathcal{L}^{\prime} \otimes \mathcal{L}^{-1} \in P_{2}$ and we are done.

We can now characterise the sections of the Jacobian surface as follows.

Lemma 2.4. Let $X$ be a non-Kähler elliptic surface. Then, any section $\Sigma$ of the Jacobian surface $J(X)$ of $X$ has trivial self-intersection. Furthermore, if $\mathcal{L}$ is any line bundle on $X$ corresponding to the section $\Sigma$ of $J(X)$, then

$$
\Sigma \cdot B_{0}=-c_{1}^{2}(\mathcal{L}) / 2
$$

where $B_{0}$ denotes the zero section of $J(X)$.

Proof. The invariants of the Jacobian surface $J(X)=B \times T^{*}$ are

$$
p_{g}(J(X))=g, q(J(X))=g+1, \text { and } K_{J(X)}=p_{1}^{*} K_{B},
$$

where $g$ is the genus of the curve $B$; the adjunction formula gives $\Sigma^{2}=0$. Let $\hat{c}_{1}$ be the class of $c_{1}(\mathcal{L})$ in $N S(X) / \operatorname{Tors}(N S(X)) \cong \operatorname{Hom}\left(J_{B}, T^{*}\right)$. As in the proof of Lemma 2.1 we can then think of $\hat{c}_{1}$ as being a covering map $\hat{c}_{1}: B \rightarrow T^{*}$ of degree $-c_{1}^{2}(\mathcal{L}) / 2$; since the degree of $\hat{c}_{1}$ is also equal to $\Sigma \cdot B_{0}$, the lemma follows.

We end the section by giving a description of torsion line bundles on a principal elliptic bundle $X \stackrel{\pi}{\rightarrow} B$; the surface is now isomorphic to a quotient of the form

$$
X=\Theta^{*} /\langle\tau\rangle,
$$

where $\Theta$ is a line bundle on $B$ with positive Chern class $d, \Theta^{*}$ is the complement of the zero section in the total space of $\Theta$, and $\langle\tau\rangle$ is the multiplicative cyclic group generated by a fixed complex number $\tau$, with $|\tau|$ greater than 1 . The standard fibre of this bundle is

$$
T \cong \mathbb{C}^{*} /\langle\tau\rangle \cong \mathbb{C} /(2 \pi i \mathbb{Z}+\ln (\tau) \mathbb{Z})
$$

(We assume $d$ to be positive so that the surface $X$ is non-Kählerian.)

The set of all holomorphic line bundles on $X$ with trivial Chern class is given by the zero component of the Picard group $\operatorname{Pic}^{0}(X)$. Referring to Proposition 1.6 in T], one has

$$
\operatorname{Pic}^{0}(X) \cong \operatorname{Pic}^{0}(B) \times \mathbb{C}^{*}
$$

Any line bundle in $\operatorname{Pic}^{0}(X)$ is therefore of the form $H \otimes L_{\alpha}$, where $H$ is the pullback to $X$ of an element of $\operatorname{Pic}^{0}(B)$ and $L_{\alpha}$ is the line bundle corresponding to the constant automorphy factor $\alpha \in \mathbb{C}^{*}$. We illustrate this by constructing the restriction of the universal (Poincaré) line bundle $\mathcal{U}$ over $X \times \operatorname{Pic}^{0}(X)$ to

$$
X \times \mathbb{C}^{*}:=X \times\{0\} \times \mathbb{C}^{*} .
$$


One starts with a trivial line bundle $\overline{\mathbb{C}}$ on $\Theta^{*} \times \mathbb{C}^{*}$ and applies to it the following $\mathbb{Z}$-action

$$
\begin{aligned}
\Theta^{*} \times \mathbb{C}^{*} \times \mathbb{Z} & \longrightarrow \Theta^{*} \times \mathbb{C}^{*} \\
(z, \alpha, n) & \longmapsto\left(\tau^{n} z, \alpha\right) .
\end{aligned}
$$

Since this action is trivial on $\mathbb{C}^{*}$, the Poincaré line bundle $\mathcal{U}$ is obtained by identifying $s \in \overline{\mathbb{C}}_{(z, \alpha)}$ with $\alpha s \in \overline{\mathbb{C}}_{(\tau z, \alpha)}$.

Notation. In the remainder, we shall denote by $L_{\alpha}$ the line bundle corresponding to the automorphy factor $\alpha \in \mathbb{C}^{*}$.

Remark. Although the line bundle $L_{\tau^{m}}$ is trivial over the fibres of $\pi$, one cannot define an action of $\mathbb{Z}$ on $\mathbb{C}^{*}$ that leaves the restriction of the Poincaré line bundle $\mathcal{U}$ to $X \times \mathbb{C}^{*}$ invariant. Indeed, if $\mathbb{Z}$ acts on $\mathbb{C}^{*}$, then multiplication by $\tau$ is defined on the fibres of $\overline{\mathbb{C}}$ by

$$
\begin{aligned}
& \tau: \Theta^{*} \times \mathbb{C}^{*} \times \mathbb{C} \quad \longrightarrow \quad \Theta^{*} \times \mathbb{C}^{*} \times \mathbb{C} \\
& (z, \alpha, t) \longmapsto(\tau z, \tau \alpha, \alpha t) .
\end{aligned}
$$

On the surface $X, z$ and $\tau z$ define the same point $x$. However, (2.5) indicates that $\tau$ sends $\mathcal{U}_{(x, \alpha)}$ to $\mathcal{U}_{(x, \tau \alpha)} \otimes L_{\tau^{-1}, x}$. Hence, the Poincaré line bundle is not invariant under such an action.

\section{HolOMORPHIC VECTOR BUNDLES}

Consider a pair $\left(c_{1}, c_{2}\right)$ in $N S(X) \times \mathbb{Z}$. Its corresponding discriminant is then given by

$$
\Delta\left(2, c_{1}, c_{2}\right):=\frac{1}{2}\left(c_{2}-\frac{c_{1}^{2}}{4}\right) \geq 0 .
$$

Let $E$ be a rank 2 vector bundle over $X$, with $c_{1}(E)=c_{1}$ and $c_{2}(E)=c_{2}$. We fix the following notation:

$$
\Delta(E):=\Delta\left(2, c_{1}, c_{2}\right) \text { and } n_{E}:=-c h_{2}(E)
$$

where $c_{2}(E)=c_{1}^{2} / 2-c_{2}$ is the second Chern character of $E$.

Remark 3.1. Referring to Lemma 2.1] if $\Delta\left(2, c_{1}, c_{2}\right) \geq 0$, then $n_{E} \geq 0$.

To study bundles on $X$, one of our main tools will be restriction of the bundle to the smooth fibres $\pi^{-1}(b) \cong T$ of the fibration $\pi: X \rightarrow B$. Since the restriction of any bundle on $X$ to a fibre $T$ has first Chern class zero, we consider $E$ as family of degree zero bundles over the elliptic curve $T$, parametrised by $B$. Given a rank two bundle over $X$, its restriction to a generic fibre of $\pi$ is semistable. More precisely, we have:

Proposition 3.2. Let $E$ be a rank 2 holomorphic vector bundle over $X$. Then, $\left.E\right|_{\pi^{-1}(b)}$ is unstable on at most an isolated set of points $b \in B$.

Proof. Suppose that $b \in B$ is a point such that $\left.E\right|_{\pi^{-1}(b)}$ is unstable, splitting as $\lambda_{b} \oplus\left(\lambda_{b}^{\prime}\right)^{*}$ for some line bundles $\lambda_{b}$ and $\lambda_{b}^{\prime}$ in $\operatorname{Pic}^{-k}(T), k>0$. Consider the elementary modification

$$
0 \rightarrow E^{\prime} \rightarrow E \rightarrow j_{*} \lambda_{b} \rightarrow 0,
$$

where $j: T_{b} \rightarrow X$ is the natural inclusion. Referring to [F2] (Chapter II, Lemma $16)$, the discriminant of $E^{\prime}$ is given by

$$
\Delta\left(E^{\prime}\right)=\Delta(E)+\frac{1}{2} j_{*} c_{1}\left(\lambda_{b}\right)
$$


furthermore,

$$
\Delta\left(E^{\prime}\right)<\Delta(E)
$$

because $\operatorname{deg}\left(\lambda_{b}\right)=-k<0$. Therefore, since the existence of $E^{\prime}$ implies that its discriminant is a non-negative number, the result follows.

Note. These isolated points are called the jumps of the bundle $E$.

3.1. The spectral curve of a rank-2 vector bundle. Let us assume for a moment that $X$ does not have multiple fibres. Choose a line bundle $L$ in $\operatorname{Pic}^{0}(X)$ such that $h^{0}\left(\pi^{-1}(b), L^{*} \otimes E\right)$ is zero, for generic $b$. The direct image sheaf $R^{1} \pi_{*}\left(L^{*} \otimes E\right)$ is therefore a torsion sheaf supported on isolated points $b$ such that $\left.E\right|_{\pi^{-1}(b)}$ is semistable and has $\left.L\right|_{\pi^{-1}(b)}$ as a subline bundle, or $\left.E\right|_{\pi^{-1}(b)}$ is unstable; consequently, if $h$ is the positive generator of $H^{2}(B, \mathbb{Z})$, then

$$
c_{1}\left(R^{1} \pi_{*}\left(L^{*} \otimes E\right)\right)=-\pi_{*}(\operatorname{ch}(E) \cdot t d(X)) \cdot t d(B)^{-1}=n_{E} h .
$$

However, since the discriminant of $E$ is a non-negative number, then so is the integer $n_{E}$ (see remark 3.1): the sheaf $R^{1} \pi_{*}\left(L^{*} \otimes E\right.$ ) is supported on $n_{E}$ points, counting multiplicity.

To obtain a complete description of the restriction of $E$ to the fibres of $\pi$, this construction must be repeated for every line bundle on $X$; this is done by taking the direct image $R^{1} \pi_{*}$ for all line bundles simultaneously. Let $\pi$ also denote the projection $\pi:=\pi \times i d: X \times \operatorname{Pic}^{0}(B) \times \mathbb{C}^{*} \rightarrow B \times \operatorname{Pic}^{0}(B) \times \mathbb{C}^{*}$, where $i d$ is the identity map on $\operatorname{Pic}^{0}(B) \times \mathbb{C}^{*}$, and let $s: X \times \operatorname{Pic}^{0}(B) \times \mathbb{C}^{*} \rightarrow X$ be the projection onto the first factor. If $\mathcal{U}$ is the universal (Poincaré) line bundle over $X \times \operatorname{Pic}^{0}(B) \times \mathbb{C}^{*}$, one defines

$$
\widetilde{\mathcal{L}}:=R^{1} \pi_{*}\left(s^{*} E \otimes \mathcal{U}\right) .
$$

This sheaf is supported on a divisor $\widetilde{S_{E}}$ that is defined with multiplicity. We have the following remarks:

- Let $H$ be the pullback to $X$ of a line bundle of degree zero on $B$. The restriction of $H$ to any fibre $T$ is then trivial, implying that the support of

$$
R^{1} \pi_{*}\left(s^{*} E \otimes \mathcal{U} \otimes H\right)
$$

is also $\widetilde{S_{E}}$. We can therefore restrict the above construction to $X \times \mathbb{C}^{*}:=$ $X \times\{0\} \times \mathbb{C}^{*}$. In the remainder, we will use the same notation for this restriction.

- Consider the $\mathbb{Z}$-action on $B \times \mathbb{C}^{*}$ induced from the one on $X \times \mathbb{C}^{*}$. For any $(b, \alpha)$ in $B \times \mathbb{C}^{*}$, multiplication by $\tau$ sends the stalk $\widetilde{\mathcal{L}}_{(x, \alpha)}$ to $\widetilde{\mathcal{L}}_{(x, \tau \alpha)} \otimes L_{\tau^{-1}, x}$, leaving the support of $\widetilde{\mathcal{L}}$ unchanged.

By the above remarks, since the quotient $\mathbb{C}^{*} /\langle\tau\rangle$ of $\mathbb{C}^{*}$ by the $\mathbb{Z}$-action is isomorphic to $T^{*}$, the support $\widetilde{S_{E}}$ of $\widetilde{\mathcal{L}}$ descends to a divisor $S_{E}$ in $J(X)=B \times T^{*}$ of the form

$$
S_{E}:=\left(\sum_{i=1}^{k}\left\{x_{i}\right\} \times T^{*}\right)+\bar{C},
$$

where $\bar{C}$ is a bisection of $J(X)$ (that is, $S_{E} \cdot T^{*}=2$ for any fibre $T^{*}$ of $J(X)$ ) and $x_{1}, \cdots, x_{k}$ are points (counted with multiplicities) in $B$ that correspond to the jumps of $E$. 
If the fibration $\pi$ has multiple fibres, the spectral cover of a bundle $E$ on $X$ is then constructed as follows. Referring to the proof of Lemma 2.2 there exists a principal $T$-bundle $\pi^{\prime}: X^{\prime} \rightarrow B^{\prime}$ over an $m$-cyclic covering $\varepsilon: B^{\prime} \rightarrow B$. Note that the map $\varepsilon$ induces natural $m$-cyclic coverings $\psi: X^{\prime} \rightarrow X$ and $J\left(X^{\prime}\right) \rightarrow J(X)$. By replacing $X$ with $X^{\prime}$ (which has no multiple fibres) in the above construction, we obtain the a spectral cover $S_{\psi^{*} E}$ of $\psi^{*} E$ as a divisor in $J\left(X^{\prime}\right)$. We define the spectral cover $S_{E}$ of $E$ as the projection of $S_{\psi^{*} E}$ in $J(X)$; one easily sees that $S_{E}$ does indeed give the isomorphism type of $E$ over each smooth fibre of $\pi$.

Remark. The above construction can be defined for any rank- $r$ vector bundle. In particular, for a line bundle, the spectral cover corresponds to the section of the Jacobian surface $J(X)$ defined in section 2 .

3.2. The graph of a rank-2 vector bundle. Let $\delta$ be the determinant line bundle of $E$. It then defines the following involution on the relative Jacobian $J(X)=B \times T^{*}$ of $X$ :

$$
\begin{aligned}
i_{\delta}: J(X) & \rightarrow J(X) \\
(b, \lambda) & \mapsto\left(b, \delta_{b} \otimes \lambda^{-1}\right),
\end{aligned}
$$

where $\delta_{b}$ denotes the restriction of $\delta$ to the fibre $T_{b}=\pi^{-1}(b)$. For fixed point $b$ in $B$, the involution induced on the corresponding fibre of $p_{1}: J(X) \rightarrow B$ has four fixed points (the solutions of $\lambda^{2}=\delta_{b}$ ). Taking the quotient of $J(X)$ by this involution, each fibre of $p_{1}$ becomes $T^{*} / i_{\delta} \cong \mathbb{P}^{1}$ and the quotient $J(X) / i_{\delta}$ is isomorphic to a ruled surface $\mathbb{F}_{\delta}$ over $B$. Let $\eta: J(X) \rightarrow \mathbb{F}_{\delta}$ be the canonical map. By construction, the spectral curve $S_{E}$ associated to $E$ is invariant under the involution $i_{\delta}$ and descends to the quotient $\mathbb{F}_{\delta}$; it can therefore be considered as the pullback via $\eta$ of a divisor on $\mathbb{F}_{\delta}$ of the form

$$
\mathcal{G}_{E}:=\sum_{i=1}^{k} f_{i}+A,
$$

where $f_{i}$ is the fibre of the ruled surface $\mathbb{F}_{\delta}$ over the point $x_{i}$ and $A$ is a section of the ruling such that $\eta^{*} A=\bar{C}$. The divisor $\mathcal{G}_{E}$ is called the graph of the bundle $E$. We finish by noting that, although the section $A$ is a smooth curve on $\mathbb{F}_{\delta}$, its pullback need not be smooth: it may be reducible or multiple with multiplicity 2 .

Remark. If $\delta$ is the pullback of a line bundle on $B$, then its restriction to any fibre of $\pi$ is trivial and the induced involution $i_{\delta}$ is given by $(b, \lambda) \mapsto\left(b, \lambda^{-1}\right)$; in this case, we have $\left(B \times T^{*}\right) / i_{\delta}=B \times \mathbb{P}^{1}$. Furthermore, if there exist line bundles $a$ and $\delta^{\prime}$ on $X$ such that $\delta=a^{2} \delta^{\prime}$, then $\mathbb{F}_{\delta}$ is isomorphic to $\mathbb{F}_{\delta^{\prime}}$; indeed, the map $a: J(X) \rightarrow J(X)$ defined by $(b, \lambda) \mapsto\left(b, a_{b} \lambda\right)$ is an isomorphism of the Jacobian surface that commutes with the involutions determined by $\delta$ and $\delta^{\prime}$. In particular, if $\delta$ is an element of $2 N S(X)$, then $\delta=a^{2}$ for some line bundle on $X$ and $\mathbb{F}_{\delta}$ is isomorphic to $B \times \mathbb{P}^{1}$.

For any $c_{1}$ in $N S(X)$, choose a line bundle $\delta$ on $X$ such that

$$
c_{1}(\delta) \in c_{1}+2 N S(X)
$$

and

$$
m_{c_{1}}:=m\left(2, c_{1}\right)=-\frac{1}{2}\left(c_{1}(\delta) / 2\right)^{2}
$$


Therefore, if $\delta^{\prime}$ is any other line bundle with Chern class in $c_{1}+2 N S(X)$, it induces a ruled surface that is isomorphic to $\mathbb{F}_{\delta}$; the advantage of using this particular $\delta$ is that its Chern class has maximal self-intersection $-8 m_{c_{1}}$.

Let us now compute the invariant of the ruled surface. We begin by setting some notation. We denote $B_{0}$ the zero-section of $J(X)$ and $\Sigma_{\delta}$ the section in $J(X)$ corresponding to $\delta$; also, let $p_{1}: J(X) \rightarrow B$ be the projection onto the first factor. Consider the exact sequence

$$
0 \rightarrow \mathcal{O}_{J(X)}\left(\Sigma_{\delta}\right) \rightarrow \mathcal{O}_{J(X)}\left(B_{0}+\Sigma_{\delta}\right) \rightarrow \mathcal{O}_{B_{0}}\left(\Sigma_{\delta}\right) \rightarrow 0 .
$$

Pushing down to $B$, we obtain a new exact sequence

$$
0 \rightarrow \mathcal{O}_{B} \rightarrow V_{\delta} \rightarrow L \rightarrow 0
$$

where

$$
V_{\delta}:=p_{1 *}\left(\mathcal{O}_{J(X)}\left(B_{0}+\Sigma_{\delta}\right)\right)
$$

is a rank-2 vector bundle on the curve $B$ and

$$
L:=p_{1 *}\left(\mathcal{O}_{B_{0}}\left(\Sigma_{\delta}\right)\right)
$$

is a line bundle of degree $4 m_{c_{1}}$ on $B$, given by the effective divisor $q_{1}+\cdots+q_{4 m_{c_{1}}}$ that corresponds to the projection onto $B$ of the intersection points $B_{0} \cap \Sigma_{\delta}$ (counted with multiplicity). Note that $\mathbb{F}_{\delta}=\mathbb{P}\left(V_{\delta}\right)$. Given the above notation, we have the following result.

Lemma 3.5. Let $d$ be the maximal degree of a subline bundle of $V_{\delta}$; it is then a non-negative integer that satisfies the inequality

$$
\max \left\{0,2 m_{c_{1}}-g / 2\right\} \leq d \leq 2 m_{c_{1}} .
$$

Moreover, the invariant of the ruled surface $\mathbb{F}_{\delta}=\mathbb{P}\left(V_{\delta}\right)$ is

$$
e=2 d-4 m_{c_{1}} .
$$

Proof. The invariant $e$ of the ruled surface is given by

$$
e=\max \left\{2 \operatorname{deg} \lambda-\operatorname{deg} V_{\delta}: \text { there exists a nonzero map } \lambda \rightarrow V_{\delta}\right\},
$$

where $\lambda$ is a line bundle on $B$ (see, for example, [F2]). Therefore, if $d$ is the maximal degree of a subline bundle of $V_{\delta}$, we have

$$
e=2 d-\operatorname{deg} V_{\delta}=2 d-4 m_{c_{1}} .
$$

Note that, since $\mathcal{O}$ is a subline bundle of $V_{\delta}$ (see (3.4) ), the integer $d$ is non-negative. To determine the bounds of $d$, we have to verify that

$$
-g \leq e \leq 0 .
$$

The left-hand inequality follows from a theorem of Segre-Nagata [F2] hence, there only remains to show that $e$ is less than or equal to zero.

Let $A$ be a section of the ruled surface $\mathbb{F}_{\delta}$; the pullback $\eta^{*} A$ is therefore a bisection of $J(X)$. If it is reducible, then its two components are sections $C_{1}$ and $C_{2}$ of $J(X)$, giving

$$
2 A^{2}=\left(\eta^{*} A\right)^{2}=\left(C_{1}+C_{2}\right)^{2}=2 C_{1} \cdot C_{2} \geq 0 .
$$

If the bisection $\bar{C}=\eta^{*} A$ is instead irreducible, we consider its normalization $C \rightarrow \bar{C}$ and let $\gamma: C \rightarrow B$ be the two-to-one map induced by $C \rightarrow \bar{C} \subset J(X)$. Note that the natural map $C \rightarrow J(X) \times{ }_{B} C$ gives a section $C_{1}$ of the surface $C \times T^{*} \rightarrow C$; moreover, if we denote by $\tilde{\gamma}: C \times T^{*} \rightarrow J(X)$ the two-to-one map induced by $\gamma$, 
then the pullback $\tilde{\gamma}^{*}(\bar{C})$ is reducible, with components $C_{1}$ and $C_{2}$, where $C_{2}$ is also section of $C \times T^{*} \rightarrow C$, and we have

$$
4 A^{2}=\left(\tilde{\gamma}^{*}(\bar{C})\right)^{2}=\left(C_{1}+C_{2}\right)^{2}=2 C_{1} \cdot C_{2} \geq 0 .
$$

Therefore, since

$$
e=-\min \left\{A^{2} \mid A \text { section of } \mathbb{F}_{\delta}\right\}
$$

(see [F2], Proposition 12, Chapter 5), it follows that $e$ is non-positive.

Remark. For a generic curve $B$ of genus greater than 1, the Neron-Severi group of an elliptic surface $X$ over $B$ is trivial and the ruled surface is $B \times \mathbb{P}^{1}$ for any $\delta$ in $\operatorname{Pic}(X)$. Moreover, this is always true if $B$ is rational: the sections of the ruled surface are given by rational maps $\mathbb{P}^{1} \rightarrow \mathbb{P}^{1}$ and the irreducible bisections of $J(X)$ are the pullbacks to $J(X)$ of non-constant rational maps (for details, see [Mo]).

We finish this section by determining the genus of irreducible bisections.

Lemma 3.6. If the spectral cover of the bundle $E$ is a smooth irreducible bisection $\bar{C}$ of $J(X)$, then its genus is given by

$$
g(\bar{C})=4 \Delta(E)+2 g-1
$$

where $g$ is the genus of $B$.

Proof. We begin by noting that the pushforward $A_{0}:=\eta_{*}\left(B_{0}\right)$ of the zero section of $J(X)$ is a section of the ruled surface $\mathbb{F}_{\delta}$ whose pullback $\eta^{*} A_{0}$ to $J(X)$ is the reducible bisection $B_{0}+\Sigma_{\delta}$; consequently, it has self-intersection $A_{0}^{2}=-c_{1}^{2} / 2$. We now describe the ramification and branching divisors of $\eta$. Let $R$ be the ramification divisor in $J(X)$, defined as the fixed point set of $\eta$; referring to Lemma 2.4 we have

$$
R \cdot B_{0}=\#\left\{(b, t): \delta_{b}=\mathcal{O}_{T}\right\}=\Sigma_{\delta} \cdot B_{0}=-c_{1}^{2} / 2 .
$$

The branching divisor $G$ is a 4 -section of $\mathbb{F}_{\delta}$ such that $\eta^{*} G=2 R$; since

$$
G \cdot A_{0}=G \cdot \eta_{*}\left(B_{0}\right)=\eta_{*}\left(\eta^{*} G \cdot B_{0}\right)=-c_{1}^{2},
$$

it is equivalent to a divisor of the form $4 A_{0}+\mathfrak{b} f$, where $\mathfrak{b}$ is a divisor on $B$ of degree $c_{1}^{2}$ and $f$ is a fibre of the ruled surface.

Let $A$ be the graph of the bundle $E$, that is, the section of $\mathbb{F}_{\delta}$ such that $\bar{C}=\eta^{*} A$. If we write $A \sim A_{0}+\mathfrak{b}^{\prime} f$, for some divisor $\mathfrak{b}^{\prime}$ on $B$, then $\bar{C} \sim\left(B_{0}+\Sigma_{\delta}\right)+\mathfrak{b}^{\prime} T^{*}$, where $\mathfrak{b}^{\prime}$ also denotes the pullback of the divisor to $J(X)$, and the intersection number $\bar{C} \cdot B_{0}$ is equal to $-c_{1}^{2} / 2+\operatorname{deg} \mathfrak{b}^{\prime}$. Recall that $\bar{C} \cdot B_{0}$ is, by construction, the number of points (counted with multiplicity) in the support of the torsion sheaf $R^{1} \pi_{*}\left(E \otimes \mathcal{O}_{X}\right)$, which is equal to $n_{E}=c_{2}-c_{1}^{2} / 2$ (see section 3); therefore, we have $\operatorname{deg} \mathfrak{b}^{\prime}=c_{2}$. Hence, the smooth bisection $\bar{C}$ is a double cover of $B$ of branching order $G \cdot A=4 c_{2}-c_{1}^{2}$ and (3.7) follows by the Hurwitz formula.

\section{Existence theOREMS}

Let $E$ be a holomorphic rank-2 vector bundle on the non-Kähler elliptic surface $X$ with determinant line bundle $\delta$ and Chern classes $c_{1}$ and $c_{2}$. If we denote

$$
\Delta(E):=\Delta\left(2, c_{1}, c_{2}\right)
$$

the discriminant of $E$, then a well-known result states that $\Delta(E)$ cannot be negative $\mathrm{BaL}, \mathrm{ElFo}, \mathrm{BrF}, \mathrm{Br}$, LeP. 
4.1. Rank-2 vector bundle as extensions. By using Lemma 2.2 Proposition 2.3 and Lemma 2.4 one obtains the following result, whose proof is similar to that of Theorem 1.3, Chapter VII, [FM]:

Theorem 4.1. Let $\pi: X \rightarrow B$ be a non-Kähler elliptic surface and $E$ be a holomorphic rank-2 vector bundle on $X$ with determinant line bundle $\delta$. Then $E$ satisfies one of the following two cases:

(A) There exists a line bundle $\mathcal{D}$ on $X$ and a locally complete intersection $Z$ of codimension 2 in $X$ such that $E$ is given by an extension

$$
0 \rightarrow \mathcal{D} \rightarrow E \rightarrow \delta \otimes \mathcal{D}^{-1} \otimes I_{Z} \rightarrow 0 .
$$

In fact, $Z$ is the set of points (counted with multiplicity) corresponding to the fibres of $\pi$ over which the bundle $E$ is unstable. Moreover, we have

$$
\Delta(E)=\frac{1}{8} \bar{C}^{2}+\frac{1}{2} \ell(Z) .
$$

(B) There exists: (i) a smooth irreducible curve $C$ and a birational map $C \rightarrow$ $\bar{C} \subset J(X)$, where $\bar{C}$ is a bisection that is invariant under the involution $i_{\delta}$ on $J(X)$ defined by the line bundle $\delta$;

(ii) a line bundle $\tilde{\mathcal{D}}$ on the normalisation $W$ of $X \times_{B} C$, whose restriction to a smooth fibre of $W \rightarrow C$ is the same as the one induced by the section of $J(W)$ that corresponds to the map $C \rightarrow J(X)$;

(iii) a codimension 2 locally complete intersection $\tilde{Z}$ in $W$, an exact sequence

$$
0 \rightarrow \tilde{\mathcal{D}} \rightarrow \tilde{\gamma}^{*} E \rightarrow \tilde{\gamma}^{*} \delta \otimes \tilde{\mathcal{D}}^{-1} \otimes I_{\tilde{Z}} \rightarrow 0
$$

where $\tilde{\gamma}: W \rightarrow X$ is the natural map, and

$$
\Delta(E)=\frac{1}{8} \bar{C}^{2}+\frac{1}{4} \ell(\tilde{Z}) .
$$

This time, $\tilde{Z}$ is the set of points corresponding to the fibres of $W \rightarrow C$ over which the bundle $\tilde{\gamma}^{*} E$ is unstable.

Remark 4.2. Suppose that the vector bundle $E$ satisfies case (A) of Theorem 4.1 Let $\Sigma_{1}$ and $\Sigma_{2}$ be the sections of $J(X)$ determined by the line bundles $\mathcal{D}$ and $\mathcal{D} \otimes \delta$, respectively. Then, one can easily verify that $\bar{C}=\Sigma_{1}+\Sigma_{2}$, implying that the bisection associated to $E$ is reducible or a section counted with multiplicity 2 (if $\Sigma_{1}=\Sigma_{2}$ ).

We now have the following complete description of non-filtrable bundles:

Proposition 4.3. Let $E$ be any holomorphic 2-vector bundle over $X$. Suppose that the spectral cover of $E$ includes the bisection $\bar{C}$ of $J(X)$. Then $E$ is non-filtrable if and only if $\bar{C}$ is irreducible.

Proof. Suppose that there exits a line bundle $\mathcal{D}$ on $X$ that maps into $E$. After possibly tensoring $\mathcal{D}$ by the pullback of a suitable line bundle on $B$, the rank-2 bundle $E$ is then given as an extension

$$
0 \rightarrow \mathcal{D} \rightarrow E \rightarrow \mathcal{D}^{-1} \otimes \delta \otimes I_{Z} \rightarrow 0,
$$

where $Z \subset X$ is a locally complete intersection of codimension 2, that is, $E$ satisfies case (A) of Theorem 4.1 referring to remark 4.2 the bisection is then not irreducible. Conversely, suppose that the bisection is not irreducible and that $\Sigma$ is one of its components. If $\mathcal{D}$ is a line bundle on $X$ corresponding to $\Sigma$, then $\mathcal{D}$ maps non-trivially into $E$, implying that $E$ is filtrable. 
Note. A partial characterisation of non-filtrable bundles is also given in ATo.

4.2. Existence of rank-2 vector bundles. A partial converse of Theorem 4.1 is the following result:

Theorem 4.4. Let $\pi: X \rightarrow B$ be a non-Kähler elliptic surface and $\delta$ be a line bundle in Pic $(X)$. Furthermore, let $i_{\delta}: J(X) \rightarrow J(X)$ be the involution defined by $\delta$ and suppose that $\bar{C}$ is a bisection of $J(X) \rightarrow B$ that is invariant with respect to the involution $i_{\delta}$. Then, there exists a rank-2 holomorphic vector bundle $E$ on $X$ such that

$$
c_{1}(E)=c_{1}(\delta) \text { and } \Delta(E)=\frac{1}{8} \bar{C}^{2}=\frac{1}{4} A^{2},
$$

where $A$ is a section of the ruled surface $\mathbb{F}_{\delta}$ with $\eta^{*} A=\bar{C}$.

Proof. If the bisection $\bar{C}$ is reducible, then its components are sections $\Sigma_{1}$ and $\Sigma_{2}$ of $J(X)$. Let $\mathcal{D}$ be a line bundle on $X$ corresponding to $\Sigma_{1}$ (see Proposition 2.3); if $E$ is any extension of $\mathcal{D}^{-1} \otimes \delta$ by $\mathcal{D}$, then $E$ is a rank-2 vector bundle on $X$ that has determinant $\delta$ and spectral cover $\bar{C}$.

If the bisection $\bar{C}$ is irreducible, then consider its normalisation $C \rightarrow \bar{C}$ and let $\gamma: C \rightarrow B$ be the double covering induced by $C \rightarrow \bar{C} \subset J(X)$. The normalisation $W$ of the fibred product $X \times_{B} C$ is then a non-Kähler elliptic surface over $C$ with relative Jacobian $J(W)=C \times T^{*}$; furthermore, the natural two-to-one map $\tilde{\gamma}: W \rightarrow X$ induces a covering $\gamma^{\prime}: J(W) \rightarrow J(X)$. Note that the inclusion map $C \rightarrow J(X) \times{ }_{B} C$ gives a section $\Sigma_{1}$ of $J(W) \rightarrow C$; the pullback $\gamma^{\prime *} \bar{C}$ is then reducible with components $\Sigma_{1}$ and $\Sigma_{2}$, where $\Sigma_{2}$ is another section of $J(W)$. By Proposition 2.3 there exists a line bundle $\mathcal{L}$ on $W$ whose restriction to any smooth fibre $T_{c}$ of $W$ is $\Sigma_{2 c}$. Let $\mathcal{D}$ be the line bundle on $W$ satisfying the equality

$$
\mathcal{L} \cong \tilde{\gamma}^{*} \delta \otimes \mathcal{D}^{-1}
$$

and define the holomorphic rank-2 vector bundle $E$ on $X$ by

$$
E:=\tilde{\gamma}_{*}(\mathcal{L})
$$

we then have to show that $E$ has first Chern class $c_{1}(\delta)$ and discriminant $\frac{1}{8} \bar{C}^{2}$.

Let $\bar{i}_{\delta}$ be the involution on $W$ that interchanges the sheets of $\tilde{\gamma}$. If $G \subset X$ is the (smooth) branch divisor of the double covering $\tilde{\gamma}: W \rightarrow X$, then there exists a line bundle $L_{0}$ on $X$ such that $L_{0}^{2}=\mathcal{O}_{X}(G)$; moreover, by Lemma 29, Chapter 2 of [F2] or by [Br4], there is an exact sequence:

$$
0 \rightarrow \bar{i}_{\delta}^{*} \mathcal{L} \otimes \tilde{\gamma}^{*} L_{0}^{-1} \rightarrow \tilde{\gamma}^{*} \tilde{\gamma}_{*}(\mathcal{L}) \rightarrow \mathcal{L} \rightarrow 0 .
$$

Since the involution $\bar{i}_{\delta}$ on $W$ is induced by interchanging the sheets of the double cover $C \rightarrow B$, the restriction of $\bar{i}_{\delta}^{*} \mathcal{L}$ to any smooth fibre $T_{c}$ of $W$ (which is not in the ramification locus of $\tilde{\gamma}$ ) is isomorphic to the restriction of $\mathcal{D}$ to the same fibre, namely to $\Sigma_{1 c}$. From the preceding exact sequence, we obtain

$$
0 \rightarrow \mathcal{D} \otimes \mathcal{O}_{W}(F) \rightarrow \tilde{\gamma}^{*} E \rightarrow \tilde{\gamma}^{*} \delta \otimes \mathcal{D}^{-1} \rightarrow 0,
$$

where $F$ is a divisor on $W$ (hence a combination of fibres of the non-Kähler elliptic surface $W \rightarrow C)$. Referring to Theorem 4.1] we have

$$
\Delta(E)=\frac{1}{8} \bar{C}^{2}=\frac{1}{4} A^{2}
$$


where $A$ is the section of the ruled surface $\mathbb{F}_{\delta}$ defined by the bisection $\bar{C}$. By $\mathrm{ABrTo}$, we also have

$$
c_{1}(E) \equiv c_{1}(\delta) \bmod \text { Tors }(N S(X)) .
$$

To get rid of the torsion, we need to add multiples of classes of fibres. Then, as in $\mathrm{ABrTo}$, we can modify the line bundle $\mathcal{L}$, by tensoring it with line bundles of the form $\mathcal{O}_{W}\left(T_{c}\right)$ or $\mathcal{O}_{W}\left(T_{i}\right)$, and obtain the desired result

$$
c_{1}(E)=c_{1}(\delta) \text {. }
$$

Note that the discriminant remains unchanged (see the formula in $\mathrm{ABrT0}$ for the direct image of a line bundle).

The above result implies that the existence problem for vector bundles is equivalent to the existence problem of bisections of $J(X)$ that are invariant under a given involution. Let us fix an element $c_{1}$ in $N S(X)$ and a line bundle $\delta$ on $X$ such that $c_{1}(\delta) \in c_{1}+2 N S(X)$ and $m_{c_{1}}:=m\left(2, c_{1}\right)=-\frac{1}{2}\left(c_{1}(\delta) / 2\right)^{2}$. Referring to section 3.2 and Lemma 3.5 the Jacobian surface $J(X)$ of $X$ is thus endowed with an involution $i_{\delta}$ and the quotient is a ruled surface $\mathbb{F}_{\delta}$ that has a non-positive invariant $e$; moreover, there is a one-to-one correspondence between sections of $\mathbb{F}_{\delta}$ and spectral curves of rank-2 vector bundles on $X$ that have determinant $\delta$ and no jumps. Therefore, the minimum value of the discriminant of a vector bundle $E$ on $X$ with first Chern class $c_{1}$ is equal to $-e / 4$. Conversely, one can show that for any integer $c_{2}$ such that $\Delta\left(2, c_{1}, c_{2}\right)$ is greater or equal to $-e / 4$, there exists a rank-2 vector bundle on $X$ with Chern classes $c_{1}$ and $c_{2}$. We can now state the main result of the paper:

Theorem 4.5. Let $X$ be a minimal non-Kähler elliptic surface over a curve $B$ of genus $g$ and fix a pair $\left(c_{1}, c_{2}\right)$ in $N S(X) \times \mathbb{Z}$. Let $m_{c_{1}}:=m\left(2, c_{1}\right)$ and choose a line bundle $\delta$ on $X$ such that $-c_{1}^{2}(\delta) / 2$ is equal to $4 m_{c_{1}}$. Then, there exists a holomorphic rank-2 vector bundle on $X$ with Chern classes $c_{1}$ and $c_{2}$ if and only if

$$
\Delta\left(2, c_{1}, c_{2}\right) \geq\left(m_{c_{1}}-d / 2\right),
$$

where $d$ is the non-negative integer determined in Lemma 3.5. Furthermore, if

$$
\left(m_{c_{1}}-d / 2\right) \leq \Delta\left(2, c_{1}, c_{2}\right)<m_{c_{1}},
$$

then the corresponding vector bundles are non-filtrable.

Proof. Recall from Lemma 3.5 that the invariant $e$ of the ruled surface is equal to $2 d-4 m_{c_{1}}$. Let $\Delta_{0}:=-e / 4=m_{c_{1}}-d / 2$ and consider $\Delta:=\Delta\left(2, c_{1}, c_{2}\right) \geq \Delta_{0}$; note that $k=2\left(\Delta-\Delta_{0}\right) \geq 0$ is an integer. It is sufficient to prove the existence of a holomorphic rank-2 vector bundle $E$ with first Chern class $c_{1}(\delta)$ and discriminant $\Delta$. Let $\bar{C}_{0}$ be a bisection of $J(X)$ of minimal self-intersection $8 \Delta_{0}$. If $k=0$, choose a holomorphic rank- 2 vector bundle $E_{0}$ corresponding to $\bar{C}_{0}$, for example, any bundle determined by Theorem 4.4

For $k>0$, choose a smooth fibre $T:=\pi^{-1}(b)$ of $\pi$, with $b \in B$, such that if the bisection $\bar{C}_{0}$ is irreducible, then the double cover $\bar{C}_{0} \rightarrow B$ does not have a branch point over $b$. Set $\delta^{\prime}:=\delta \otimes \mathcal{O}_{X}(k T)$. The line bundles $\delta$ and $\delta^{\prime}$ then both correspond to the same section in $J(X)$, inducing isomorphic ruled surfaces $\mathbb{F}_{\delta^{\prime}}$ and $\mathbb{F}_{\delta}$, respectively. Consequently, there exists a holomorphic rank-2 vector bundle $E_{0}^{\prime}$ on $X$ with first Chern class $c_{1}\left(\delta^{\prime}\right)$ and discriminant $\Delta_{0}$ that is regular on the fibre $T$ (over an elliptic curve, a bundle is said to be regular if its group of automorphisms 
is of the smallest possible dimension). Indeed, if $\bar{C}_{0}$ is reducible, then choose line bundles $L_{1}$ and $L_{2}$ on $X$ associated to the components of $\bar{C}_{0}$, with $L_{1} \otimes L_{2}=\delta^{\prime}$, and let $E_{0}^{\prime}$ be an extension of $L_{2}$ by $L_{1}$ that is regular on $T$. Moreover, if $\bar{C}_{0}$ is irreducible, then $E_{0}^{\prime}$ can be any vector bundle given by Theorem 4.4 Let $j: T \rightarrow X$ be the natural inclusion map; if $\lambda$ is a line bundle on $T$ of degree 1, then there exists a surjection $E_{0}^{\prime} \rightarrow j_{*} \lambda$. Consider the elementary modification

$$
0 \rightarrow E_{1} \rightarrow E_{0}^{\prime} \rightarrow j_{*} \lambda \rightarrow 0
$$

then, the bundle $E_{1}$ splits as $\lambda \oplus \lambda^{*}$ over $T$ and there exists a surjection $E_{1} \rightarrow j_{*} \lambda$. Hence, by performing $(k-1)$ successive elementary modifications on $E_{1}$ with respect to $j_{*} \lambda$, one obtains a holomorphic vector bundle $E$ on $X$ with first Chern class $c_{1}(\delta)$ and discriminant $\Delta$.

Remark. If the genus of the base curve $B$ is less than 2, then the statement of the theorem becomes: there exists a holomorphic rank-2 vector bundle $E$ on $X$ with Chern classes $c_{1}$ and $c_{2}$ if and only if the discriminant $\Delta\left(2, c_{1}, c_{2}\right)$ is a nonnegative number. (For an alternate proof in the case of primary Kodaira surfaces, see $\mathrm{ABrTo}$.) In contrast, if the genus of the base curve is greater 1 , there are "gaps" for the discriminant of holomorphic rank- 2 vector bundles, whenever $m_{c_{1}}$ is greater than $d / 2$; thus, the existence of holomorphic vector bundles on $X$ depends on the geometry of the base curve $B$. However, by the proof of Theorem 4.5 . once there is an irreducible bisection of $J(X)$, one can construct infinitely many non-filtrable vector bundles.

Note. Bundles with $\Delta\left(2, c_{1}, c_{2}\right)=0$ have also been studied in $\mathrm{ABr}$.

Acknowledgements The first author would like to express his gratitude to the Max Planck Institute of Mathematics for its hospitality and stimulating atmosphere; this paper was prepared during his stay at the Institute. It is a pleasure for both authors to thank Jacques Hurtubise for suggesting a link between the papers ABrTo and $\mathrm{Mo}$. The second author would like to thank Jacques Hurtubise for his generous encouragement and support during the completion of this paper. She would also like to thank Ron Donagi and Tony Pantev for valuable discussions, and the Department of Mathematics at the University of Pennsylvania for their hospitality, during the preparation of part of this article.

\section{REFERENCES}

[ABr] M. Aprodu and V. Brînzănescu, On the holomorphic rank-2 vector bundles with trivial discriminant over non-Kähler elliptic bundles, to appear in J. Math. Kyoto Univ..

[ABrTo] M. Aprodu, V. Brînzănescu, and M. Toma, Holomorphic vector bundles on primary Kodaira surfaces, Math. Z. 242 (2002) 63-73; arXiv:math.CV/9909136

[ATo] M. Aprodu and M. Toma, Une note sur les fibrés holomorphes non-filtrables, Preprint 2002.

[BaL] C. Bănică and J. Le Potier, Sur l'existence des fibrés vectoriels holomorphes sur les surfaces non-algébriques, J. Reine Angew. Math. 378 (1987) 1-31.

[BVP] W. Barth, C. Peters, and A. Van de Ven, Compact complex surfaces, Springer-Verlag, Berlin-Heidelberg-New York, 1984.

[BJPS] M. Bershadsky, A. Johansen, T. Pantev, and V. Sadov, On four-dimentional compactifications of F-theory, Nuclear Phys. B 505(1-2) (1997) 165-201.

[BH] P. J. Braam and J. Hurtubise, Instantons on Hopf surfaces and monopoles on solid tori, J. Reine Angew. Math. 400 (1989) 146-172. 
[Br1] V. Brînzănescu, Néron-Severi group for non-algebraic elliptic surfaces I: elliptic bundle case, Manuscripta Math. 79 (1993) 187-195; II: non-Kählerian case, Manuscripta Math. 84 (1994) 415-420; III, Rev. Roumaine Math. Pures Appl. 43(12) (1998) 89-95.

[Br2] V. Brînzănescu, The Picard group of a primary Kodaira surface, Math. Ann. 296 (1993) 725-738.

[Br3] V. Brînzănescu, Holomorphic vector bundles over compact complex surfaces, Lecture Notes in Mathematics 1624, Springer, 1996.

[Br4] V. Brînzănescu, Double covers and vector bundles, An. Stiint. Univ. Ovidius Constanta, Ser. Mat. 9 (2001) no 1 21-26.

[BrF] V. Brînzănescu and P. Flondor, Holomorphic 2-vector bundles on non-algebraic 2tori, J. Reine Angew. Math. 363 (1985) 47-58.

[BrMo1] V. Brînzănescu and R. Moraru, Twisted Fourier-Mukai transforms and bundles on non-Kähler elliptic surfaces, preprint arXiv:math.AG/0309031

[BrMo2] V. Brînzănescu and R. Moraru, Stable bundles on non-Kähler elliptic surfaces, preprint arXiv:math.AG/0306192

[BrU] V. Brînzănescu and K. Ueno, Néron-Severi group for torus quasi bundles over curves. Moduli of vector bundles (Sanda, 1994; Kyoto, 1994), Lecture Notes in Pure and Applied Mathematics 179, Dekker, New York, 1996, 11-32.

[D] R. Donagi, Principal bundles on elliptic fibrations, Asian J. Math. 1(2) (1997) 214223; arXiv:alg-geom/9702002

[DOPW1] R. Donagi, B. Ovrut, T. Pantev, and D. Waldram, Standard-model bundles, Adv. Theor. Math. Phys. 5(3) (2001) 563-615; arXiv:math.AG/0008010

[DOPW2] R. Donagi, B. Ovrut, T. Pantev, and D. Waldram, Standard models from heterotic M-theory, Adv. Theor. Math. Phys. 5(1) (2001) 93-137; arXiv:hep-th/0008008

[ElFo] G. Elencwajg and O. Forster, Vector bundles on manifolds without divisors and a theorem of deformation, Ann. Inst. Fourier 32(4) (1982) 25-51.

[F1] R. Friedman, Rank two vector bundles over regular elliptic surfaces, Invent. Math. 96 (1989) 283-332.

[F2] R. Friedman, Algebraic surfaces and holomorphic vector bundles, Universitext, Springer-Verlag, 1998.

[FM] R. Friedman and J. W. Morgan, Smooth Four-Manifolds and Complex Surfaces, Springer-Verlag, 1994.

[FMW] R. Friedman, J. Morgan, and E. Witten, Vector bundles over elliptic fibrations, J. Algebraic Geom. 2 (1999) 279-401; arXiv:alg-geom/9709029

[Kod] K. Kodaira, On the structure of compact complex analytic surfaces I, Amer. J. Math. 86 (1964) 751-798.

[LeP] J. Le Potier, Fibrés vectoriels sur les surfaces K3, Séminaire Lelong-DolbeaultSkoda, Lecture Notes in Mathematics 1028, Springer, Berlin, 1983.

[Mo] R. Moraru, Integrable systems associated to a Hopf surface, Canad. J. Math. 55(3) (2003) 609-635.

[S] R. L. E. Schwarzenberger, Vector bundles on algebraic surfaces, Proc. London Math. Soc. 3 (1961) 601-622.

[T] A. Teleman, Moduli spaces of stable bundles on non-Kähler elliptic fibre bundles over curves, Expo. Math. 16 (1998) 193-248.

[TTo] A. Teleman and M. Toma, Holomorphic vector bundles on non-algebraic surfaces, C. R. Acad. Sci. Paris 334 (2002) 1-6; arXiv:math.AG/0201236

[To] M. Toma, Stable bundle with small $c_{2}$ over 2-dimensional complex tori, Math. Z. 232 (1999) 511-525.

Institute of Mathematics "Simion Stoilow", Romanian Academy, P.O.Box 1-764, RO70700, Bucharest, Romania

E-mail address: Vasile.Brinzanescu@imar.ro

Department of Mathematics and Statistics, Burnside Hall, McGill University, 805 Sherbrooke Street West, Montreal, Quebec, Canada, H3A 2K6

E-mail address: moraru@math.mcgill.ca 